\title{
Ultrafast Laser Writing in Transparent Materials: from Physics to Applications
}

\author{
Peter G. Kazansky \\ Optoelectronics Research Centre, University of Southampton, SO17 1BJ, United Kingdom \\ pgk@orc.soton.ac.uk
}

\begin{abstract}
Properties of optical materials can be modified with sub-wavelength precision using intense ultrashort light pulses. New science and emerging applications of ultrafast laser writing in transparent materials are reviewed.
\end{abstract}

\author{
(C) 2012 Optical Society of America \\ OCIS codes (140.3390) Laser materials processing, (320.7120) Ultrafast phenomena
}

Progress in high power ultrashort pulse lasers has opened new frontiers in physics and technology of light-matter interactions [1]. In particular, modification of transparent materials with ultrafast lasers has recently attracted considerable interest because of its new science and relevance to a wide range of applications including laser surgery, integrated optics, microfluidics, optical data storage and three-dimensional micro- and nano-structuring $[2]$.

A key advantage of using femtosecond pulses, as opposed to longer pulses, for direct writing is that they can rapidly and precisely deposit energy in solids [3]. The processes, initiated by multiphoton ionization, exhibit a highly nonlinear dependence on the intensity of the light beam. The light is absorbed by photoelectrons and the optical excitation ends before the surrounding lattice is perturbed, what results in highly localized modification without collateral material damage.

Three different types of material modifications can be induced with ultrafast laser irradiation in the bulk of a transparent material, e.g. in silica glass with increase of fluence: an isotropic refractive index change; a form birefringence associated with self-assembled nanogratings with features smaller than $20 \mathrm{~nm}$ [4-6]; and a void. Considerable efforts have been carried out to clarify the mechanisms of material modifications, which are still not fully understood.

More recently, the self-assembled nano-structuring have been proposed for fabrication of radial, azimuthal and vortex polarization converters [7] (Fig. 1) and rewritable polarization multiplexed optical memory [8], where the information encoding is realized by means of two birefringence parameters, i.e. the slow axis orientation ( $4^{\text {th }}$ dimension) and retardance $\left(5^{\text {th }}\right.$ dimension), in addition to three spatial coordinates (Fig. 2$)$. The phase of the

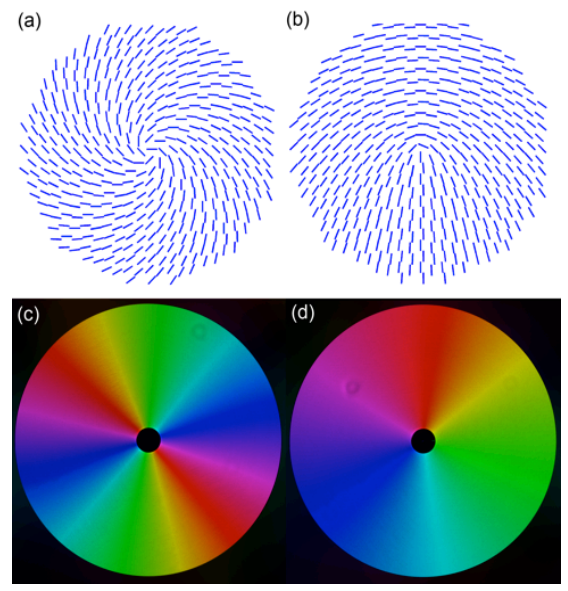

Fig. 1. Schematic drawings of nanogratings distribution in quarter- (a) and half-wave (b) polarization converters. Femtosecond laser written radial polarization converters for circular (c) and linear (d) incident polarizations. The pseudo color indicates direction of the slow axis.

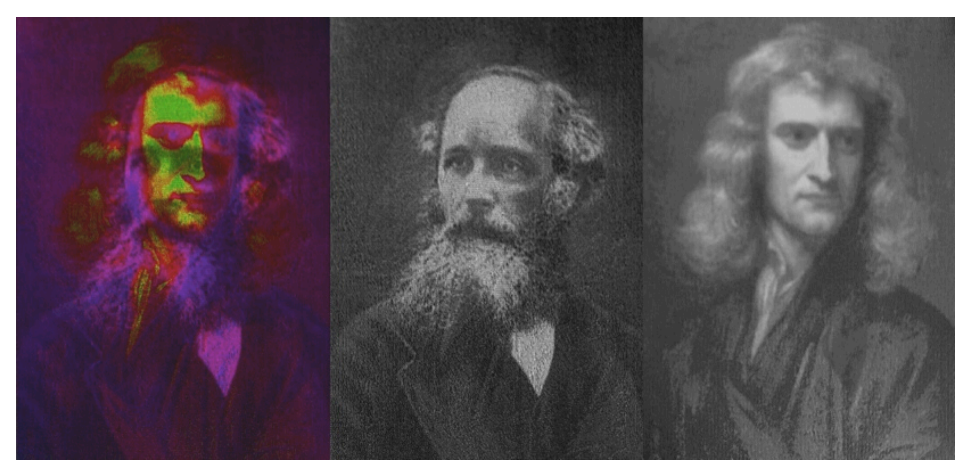

Fig. 2. Ultrafast laser optical recording via self-assembled nanograting induced birefringence in fused silica. Maxwell and Newton are recorded in one image (left, in pseudo colours), however, they can be easily decoupled as Maxwell is recorded in strength of retardance (centre) and Newton in azimuth of the slow axis (right). Size is $1.5 \times 2 \mathrm{~mm}$. 
optical beam around a point, where the intensity is zero, e.g. in vortex beam, is undefined. The tight control of nanostructures' properties through writing parameters is demonstrated implementing elements with unique optical properties, in particular Super-Structured-waveplates (S-waveplates) for uniform to radial and azimuthal polarization and vortex conversion, which can be used in material processing, microscopy, fiber optics, optical trapping and manipulation.

A remarkable effect has also been discovered, referred to as quill or calligraphic laser writing, which reveals strong dependence of material modification, in particular the self-assembled sub-wavelength structures in glass, on orientation of the writing direction relative to the direction of pulse front tilt $[9,10]$. More recently it has been demonstrated that the tip of an ultrafast laser quill has a property that is very different from an ordinary quill [11]. Specifically, the modification of glass can be controlled even in stationary conditions by the mutual orientation of light polarization azimuth and the pulse front tilt (Fig. 3). Figuratively, the polarization can be used as a

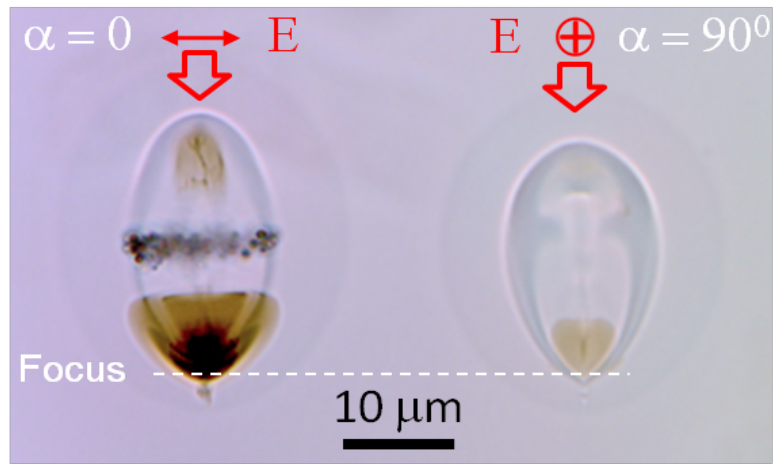

Fig. 3. Images of modified regions in glass along beam propagation direction for writing beam polarised along (left) and perpendicular (right) to the pulse intensity front normal.

sharpening blade for the ultrafast light quill. The demonstrations of self-assembled nano-structuring and employing mutual orientations of beam movement or the light polarization plane and pulse front tilt to control interaction of matter with ultrashort light pulses, open new opportunities in material processing. Another interesting phenomenon is non-reciprocal ultrafast laser writing in non-centrosymmetric medium, which contradicts to the common believe that in a homogeneous medium, the photosensitivity and corresponding lightinduced material modifications do not change on the reversal of light propagation direction [12].

\section{References}

[1] A. C. Tien, S. Backus, H. Kapteyn, M. Murnane, and G. Mourou, "Short-pulse laser damage in transparent materials as a function of pulse duration," Phys. Rev. Lett. 82, 3883 (1999).

[2] R. R. Gattas and E. Mazur, "Femtosecond laser micromachining in transparent materials," Nat. Photonics 2, 219 (2008).

[3] B. C. Stuart, M.D. Feit, A. M. Rubenchik, B. W. Shore, and M. D. Perry, "Laser-induced damage in dielectrics with nanosecond to subpicosecond pulses," Phys. Rev. Lett. 74, 2248 (1995).

[4] Y. Shimotsuma, P. G. Kazansky, J. Qiu, and K. Hirao, "Self-organized nanogratings in glass irradiated by ultrashort light pulses," Phys. Rev. Lett. 91, 247705 (2003).

[5] V. Bhardwaj, E. Simova, P. Rajeev, C. Hnatovsky, R. Taylor, D. Rayner, and P. Corkum, "Optically produced arrays of planar nanostructures inside fused silica," Phys. Rev. Lett. 96, 057404 (2006).

[6] C. Hnatovsky, V. Shvedov, W. Krolikowski, and A. Rode, "Revealing local field structure of focused ultrashort pulses," Phys. Rev. Lett.106 123901 (2011).

[7] M. Beresna, M. Gecevičius, P. G. Kazansky, and T. Gertus, "Radially polarized optical vortex converter created by femtosecond laser nanostructuring of glass," Appl. Phys. Lett. 98, 201101 (2011).

[8] Y. Shimotsuma, M. Sakakura, PG. Kazansky, M. Beresna, J. Qiu, K. Miura, and K. Hirao, "Ultrafast manipulation of selfassembled form birefringence in glass,"Adv, Materials 22 (36), 4039 (2010).

[9] P. G. Kazansky, W. Yang, E. Bricchi, J. Bovatsek, A. Arai, Y. Shimotsuma, K. Miura, and K. Hirao, "Quill" writing with ultrashort light pulses in transparent materials,” Appl. Phys. Lett. 90, 151120 (2007).

[10] D. N. Vitek, E. Block, Y. Bellouard, D. E. Adams, S. Backus, D. Kleinfeld, C. G. Durfee, and J. A. Squier, "Spatiotemporally focused femtosecond laser pulses for nonreciprocal writing in optically transparent materials," Opt. Express 18, 24673 (2010).

[11] P.G. Kazansky, Y. Shimotsuma, M. Sakakura, M. Beresna, M. Gecevicius, Yu. P. Svirko, S. Akturk, J. Qiu, K. Miura, and K. Hirao "Photosensitivity control of an isotropic medium through polarization of light pulses with tilted intensity front," Opt. Express 19, 20658 (2011)

[12] W. Yang, P. G. Kazansky, and Yu. P. Svirko, “Non-reciprocal ultrafast laser writing,” Nat. Photonics 2, 99 (2008). 